\title{
Automated Vehicle Driving using Image Processing
}

\author{
Mrs. Susmitha B C $\mathbf{C}^{\mathbf{1}}$, Karthik Sharma $\mathrm{K}^{2}$, Pavithra $\mathbf{N}^{3}$, Nandini M R ${ }^{4}$, Punyashree M C \\ Assistant Professor, ECE, GMIT, Mandya, India ${ }^{1}$ \\ $8^{\text {th }}$ Semester, ECE, GMIT, Mandya, India ${ }^{2,3,4,5}$
}

\begin{abstract}
This project focuses on control and automation of intelligent road symbol detection system for vehicles in normal environment conditions. The objective is to look for matching information or some data in the input images that are taken by the overhead mounted camera. The whole setup then filters the noise and other requirements to obtain a steady flow of information for the vehicle to be guided automatically. Here we have used python for image processing and a microcontroller interfaced with it for actual real time processing and actuation of commands.
\end{abstract}

Keywords: Intelligent, matching information, image, python.

\section{INTRODUCTION}

"The Global Status Report on Road Safety" 2015, reflects the information from 180 countries, indicates that there are around 1.25 million number of deaths due to road accidents per year. The total cost incurred does not comply well with the deteriorating Indian Economy. Here we provide with a solution to design of the automobiles involved in road accidents. Initially vehicles are invented to minimize strain on human and to increase the productivity, as the population increases many advancements where made in the vehicle in order give comfort to the people who travel in it. In the present scenario production of vehicles have increased enormously so as the user which thereby leads to heavy traffic.

Rushing around, trying to get errands done, thinking about the things to be bought from the nearest grocery store has become a part of our daily schedule. Driver error is one of the most common cause of traffic accidents, and with cell phones, in car entertainment systems, more traffic and more complicated road systems, it isn't likely to go away. With the number of accidents increasing day by day, it has become important to take over the human errors and help the mankind. All of this could come to an end with self-driving cars which just need to know the destination and then let the passengers continue with their work. This will avoid not only accidents but also bring a self-relief for minor day to day driving activities for small items.

Autonomous vehicles have been in development for over 65 years-in fact the first cruise control systems were introduced in 1948. Multiple car manufacturers estimate the first Commercial driverless cars to be released by 2020 . The promise of saving 1.2 million lives a year and solving traffic congestion problems has struck a chord with scientists, engineers, and programmers around the world. Thanks to quantum leaps made in computing technologies in the past 30 years - cheap sensing, reliable object recognition, and real-time, portable, large-scale data analysisautomated vehicles are becoming a reality. Inspired by ongoing research today from around the world, this MQP aims to imagine a divergent take on autonomous vehicle technology by challenging modern vision algorithms combined with affordable sensing technology.

The path the industry will take to evolve autonomous vehicles is generally agreed upon by large automobile manufacturers and market analysis groups alike. Several other labs have demonstrated the viability of autonomous cars in general, such as those of Google and the Autonomous Systems Laboratory at the University of California, Santa Cruz, as well as several major car manufacturers including Toyota, Nissan, Cadillac, and Audi, but many of these systems rely on expensive, bulky, and ungainly roof mounted LIDAR detectors. A unique part of the mission of this MQP is to achieve the same or better results using vision only - clearly it is possible because humans do this already.

Autonomous vehicle research has exploded in past decades, due to increased fascination with driverless vehicles and the impact they can have on society. Cars today come with options for adaptive cruise control, lane detection, and automated parallel parking - all features that rely on sensors and computing. Further advanced autonomous vehicles blend human-control with autonomous systems, and as result, have the ability to activate brakes in emergencies or alert drivers of dangers. Tracing the origins and historical discoveries of autonomous vehicle technologies leads us to the basis for the Robocart MQP. 


\section{International Journal of Innovative Research in Electrical, Electronics, Instrumentation and Control Engineering}

\section{ISO 3297:2007 Certified}

Vol. 5, Issue 6, June 2017

\section{RELATED WORKS}

The study is undertaken with the objective to detect road signs and to suggest automated driver guidance mechanism. Developing an automated driver guidance system is very important in the context of Indian road conditions. A driver finds it difficult to control the vehicle due to sudden pot holes or bumps or sudden turns where the road signs are not very prominent or missing most of the times.

Suppose if there is a system with integrated motion camera and an integrated onboard computer with the vehicle, a simple driver guidance system based on frame by frame analysis of the motion frames can be developed and there by generate the alarm signals accordingly. So that the driving can be made quite easier. Road Image analysis is very important aspect for automated driver support system. Real-time qualitative road data analysis is the corner stone for any modern transport system. So far, most of the analysis is done manually and the use of image processing techniques for qualitative analysis is still at its early stage [1].

Lane detection is one of the most challenging problems in machine vision and still has not been fully accomplished because of the highly sensitive nature of computer vision methods. Computer vision depends on various ambient factors. External illumination conditions, camera and captured image quality etc. effect machine vision performance. Lane detection faces all these challenges as well as those due to loss of visibility, types of roads, road structure, road texture and other obstacles like trees, passing vehicles and their shadows. Lane detection and its tracking is significant element for vision based driver assistance system. Main purpose of these systems is to prevent crashes due to unintended lane departure movements produced by tired drivers[2].

Driver assistance systems aim at increasing the comfort and safety of traffic participants by sensing the environment, analyzing the situation, and signalling relevant information to the driver. In order to reliably accomplish this demanding task, the information of different sensors must be evaluated and fused to obtain a suitable representation of the traffic situation. The complexity of the whole data processing architecture is determined by the actual task to which the driver assistance system is devoted. Among others, these tasks include lane departure warning, lane keeping, collision warning or avoidance, adaptive cruise control, and low-speed automation in congested traffic[3].

Build a monocular vision autonomous car prototype using Raspberry Pi as a processing chip. An HD camera along with an ultrasonic sensor is used to provide necessary data from the real world to the car. The car is capable of reaching the given destination safely and intelligently thus avoiding the risk of human errors. Many existing algorithms like lane detection, obstacle detection are combined together to provide the necessary control to the car. In robotics, obstacle avoidance is the task of satisfying the control objective subject to non-intersection or non-collision position constraints. The different hardware components and their assembly are clearly described. A novel method to determine the uneven, marked or unmarked road edges is explained in details relying upon OpenCV. Using ultrasonic sensors, the collisions with obstacles is avoided[4].

Advanced Driving Assistant Systems, intelligent and autonomous vehicles are promising solutions to enhance road safety, traffic issues and passengers comfort. Such applications require advanced computer vision algorithms that demand powerful computers with high-speed processing capabilities. Keeping intelligent vehicles on the road until its destination, in some cases, remains a great challenge, particularly when driving at high speeds. The first principle task is robust navigation, which is often based on system vision to acquire RGB images of the road for more advanced processing. The second task is the vehicle's dynamic controller according to its position, speed and direction. This paper presents an accurate and efficient road boundaries and painted lines detection algorithm for intelligent and autonomous vehicle. It combines Hough Transform to initialize the algorithm at each time needed, and Canny edges detector, least-square method and Kalman filter to minimize the adaptive region of interest, predict the future road boundaries location and lines parameters[5].

Autonomous driving cars have become a trend in the vehicle industry. Numerous driver assistance systems (DAS) have been introduced to support these automatic cars. Among these DAS methods, traffic light detection (TLD) plays a significant role. This paper proposes a method to detect traffic lights (TLs) using color density identification(CD). The system receives an RGB image as an input and produces the traffic light state (red, yellow, green or no Signal) of the scene. The algorithm has three stages: clustering, filtering, and state identification.

HSV thresholding, geometrical information, and color density of an image are used to detect traffic lights. The color density method recognizes the color of an object by comparing the densities of each color belonging to the object. The method is robust, only requires RGB images and has a high detection rate[6]. 
ISO 3297:2007 Certified

Vol. 5, Issue 6, June 2017

\section{METHODOLOGY}

\section{INPUT UNIT}

A Raspberry Pi board (model B+), attached with a pi camera module and an HC-SR04 ultrasonic sensor is used to collect input data. Two client programs run on Raspberry Pi for streaming color video and ultrasonic sensor data to the computer via local Wi-Fi connection. In order to achieve low latency video streaming, video is scaled down to QVGA $(320 \times 240)$ resolution.

\section{PROCESSING UNIT}

The processing unit (computer) handles multiple tasks: receiving data from Raspberry Pi, neural network training and prediction(steering), object detection(stop sign and traffic light), distance measurement(monocular vision), and sending instructions to Raspberry pi GPIO pins.

\section{TCP Server}

A multithread TCP server program runs on the computer to receive streamed image frames and ultrasonic data from the Raspberry Pi. Image frames are converted to gray scale and are decoded into numpy arrays.

\section{Neural Network}

One advantage of using neural network is that once the network is trained, it only needs to load trained parameters afterwards, thus prediction can be very fast. Only lower half of the input image is used for training and prediction purposes. There are 38,400 (320×120) nodes in the input layer and 32 nodes in the hidden layer. The number of nodes in the hidden layer is chosen fairly arbitrary. There are four nodes in the output layer where each node corresponds to the steering control instructions: left, right, forward and reverse respectively (though reverse is not used anywhere in this project, it's still included in the output layer).
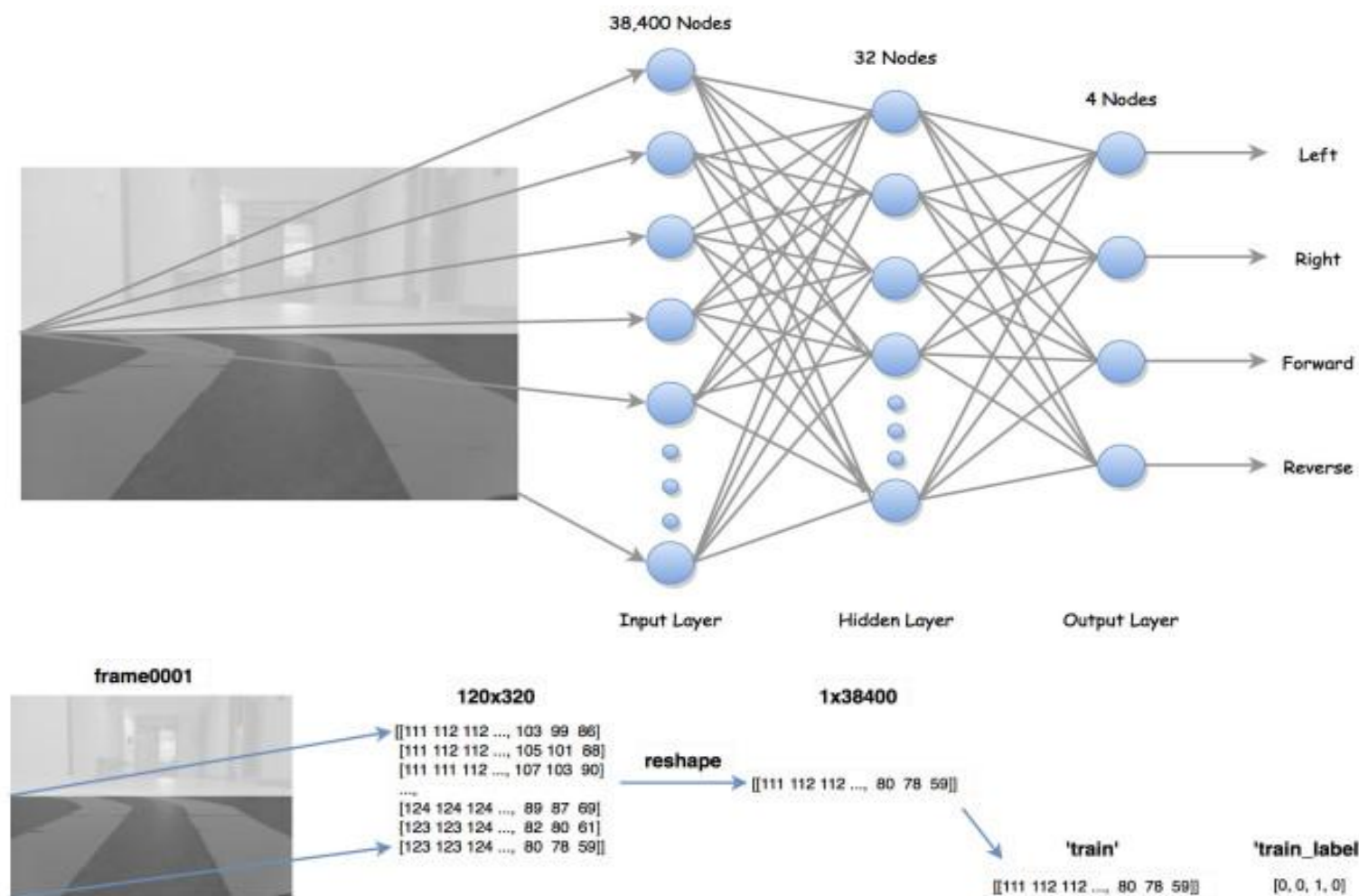

Hidden Loyer

Output Layer

trame 000

$1111112112 \ldots, 10399 \mathrm{Be}$ [111 $112112 \ldots, 10510186$

[124 $124124 \ldots 898769$ [123 $123124 \ldots, 828061$

$\stackrel{\text { reshape }}{\longrightarrow}$ II111 $112112, \ldots, 8078$ 59!


$1 \times 4$

II111 $112112 \ldots 8078$ 59]

$[0,0,1,0]$

left $=[1,0,0,0]$

right $=[0,1,0,0]$

forward $=[0,0,1,0]$

reverse $=[0,0,0,1]$ (frame0001)

trame000e

trame000s

trame0004 
Above shows the training data collection process. First each frame is cropped and converted to a numpy array. Then the train image is paired with train label (human input). Finally, all paired image data and labels are saved into a npz file. The neural network is trained in OpenCV using back propagation method. Once training is done, weights are saved into a xml file. To generate predictions, the same neural network is constructed and loaded with the trained xml file.

\section{Object Detection}

This project adapted the shape-based approach and used Haar feature-based cascade classifiers for object detection. Since each object requires its own classifier and follows the same process in training and detection, this project only focused on stop sign and traffic light detection.

OpenCV provides a trainer as well as detector. Positive samples (contain target object) were acquired using a cell phone, and were cropped that only desired object is visible. Negative samples (without target object), on the other hand, were collected randomly. In particular, traffic light positive samples contains equal number of red traffic lights and green traffic light. The same negative sample dataset was used for both stop sign and traffic light training. Below shows some positive and negative samples used in this project.
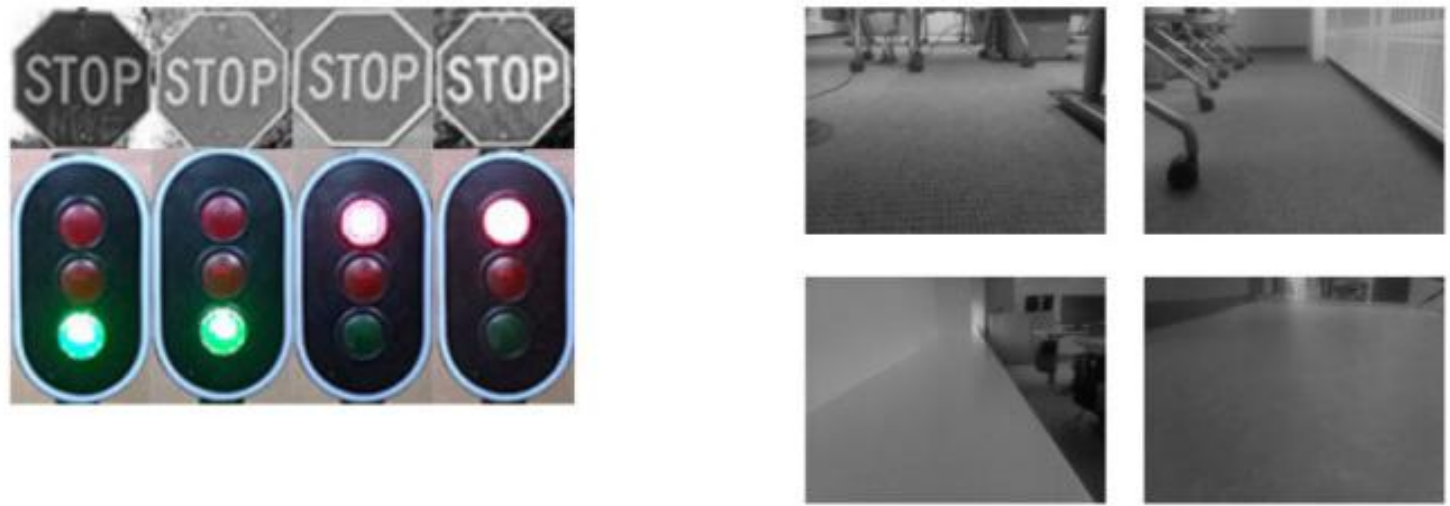

Type Number of positive sample Number of negative sample Sample size (pixels)

\begin{tabular}{llll|}
\hline Stop sign & 20 & 400 & $25 \times 25$ \\
\hline Traffic light & 26 & 400 & $25 \times 45$ \\
\hline
\end{tabular}

To recognize different states of the traffic light (red, green), some image processing is needed beyond detection. Flowchart below summarizes the traffic light recognition process.



Firstly, trained cascade classifier is used to detect traffic light. The bounding box is considered as a region of interest (ROI). Secondly, Gaussian blur is applied inside the ROI to reduce noises. Thirdly, find the brightest point in the ROI. Finally, red or green states are determined simply based on the position of the brightest spot in the ROI.

\section{IV.RESULTS}

Prediction on the testing samples returns an accuracy of $85 \%$ compared to the accuracy of $96 \%$ that the training samples returns. In actual driving situation, predictions are generated about 10 times a second streaming rate roughly $10 \mathrm{frames} / \mathrm{sec}$. 
Haar features by nature are rotation sensitive. In this project, however, rotation is not a concern as both the stop sign and the traffic lights are fixed objects which is also a general case in real world environment.
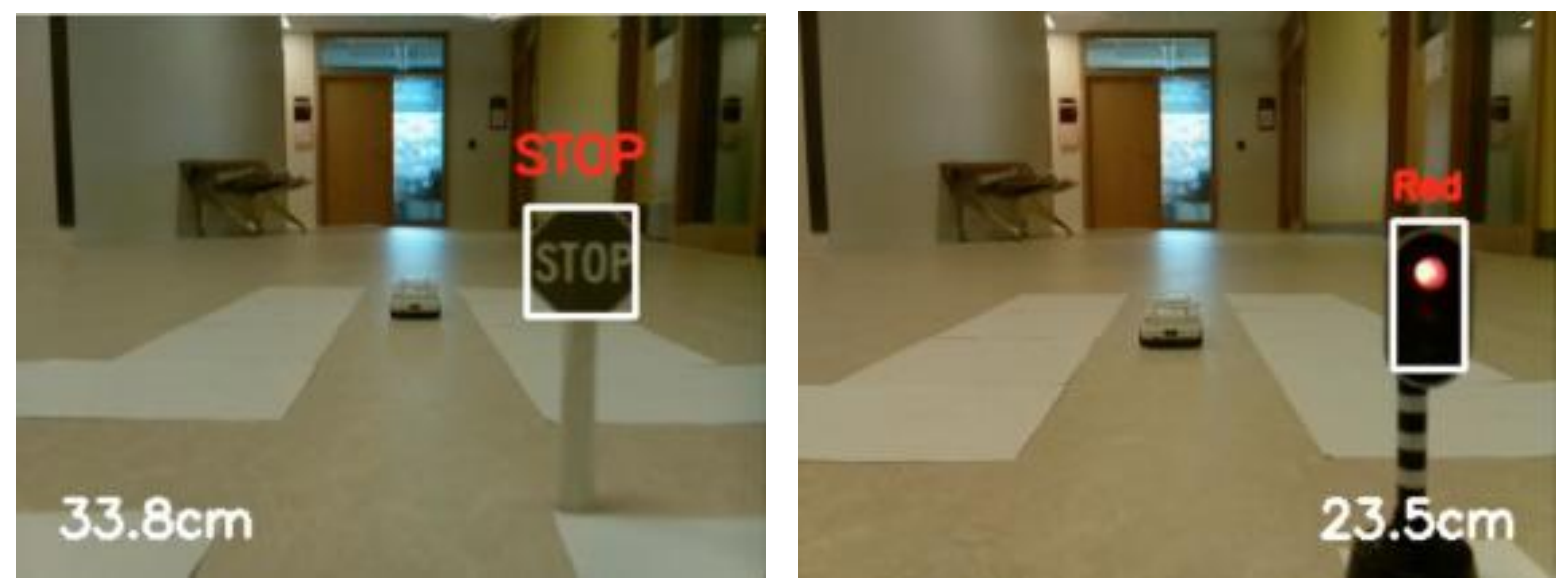

For distance measurement aspect, the ultrasonic sensor is only used to determine the distance to an obstacle in front of the RC car and provides accurate results when taking proper sensing angle and surface condition into considerations. On the other hand, Pi camera provides "good enough" measurement results. In fact, as long as we know the corresponding number to the actual distance, we know when to stop the RC car.

Overall, the RC car could successfully navigate on the track with the ability to avoid front collision, and respond to traffic light and stop sign accordingly.

\section{CONCLUSION}

The image processing algorithms used here have found a lot of practical applications and it is still one of the most extensively researched areas. A step has been taken to improve the current road traffic management scheme and provide with much cheaper and efficient results than the existing know-how. These results or their underlying principles can be deployed in different purviews like disaster mitigation management, defense etc. This algorithm can be further improved by training our dataset using machine learning algorithms which can lead to much better results with better efficiency due to reduction in processing time and output deliverance and with an up to date technology.

\section{FUTURE SCOPE}

Technologies like corporative driving and inter-vehicle communication can be further included. In order to enhance the efficiency grid cameras and night vision cameras can be installed to achieve a clear and better resolution of images in case of fog, rainfall, night times and other such undesirable environmental conditions. A real time system can be employed which has the ability to detect multiple road symbols and respond accordingly.

\section{REFERENCES}

[1]. Pranjali Ulhe, Samta Gawande, Kajol Taksande, Ankita Bakade, Image processing on Road detection, Proceedings of 19th IRF International Conference, 1st February 2015.

[2]. Sunil Kumar Vishwakarma, Akash, Divakar Singh Yadav, Analysis of Lane Detection Techniques using OpenCV”, 2015 IEEE.

[3]. Thomas Bucher, Cristobal Curio, Johann Edelbrunner, Christian Igel, Image Processing and Behavior Planning for Intelligent Vehicles, IEEE Transactions On Industrial Electronics, VOL. 50, NO. 1, February 2003.

[4]. Gurjashan Singh Pannu, Mohammad Dawud Ansari, Pritha Gupta, Design and Implementation of Autonomous Car using Raspberry Pi, International Journal of Computer Applications (0975 - 8887) Volume 113 - No. 9, March 2015

[5]. Farid Bounini, Denis Gingras, Vincent Lapointe, Herve Pollart, Autonomous Vehicle And Real Time Road Lanes Detection And Tracking, 2015 IEEE.

[6]. Tai Huu - Phuong Tran, Cuong Cao Pham, Tien Phuoc Nguyen, Tin Trung Duong, and Jae Wook Jeon, Real-Time Traffic Light Detection Using Color Density, 2016 IEEE International Conference on Consumer Electronics-Asia (ICCE-Asia).

[7]. Joel C. McCall \&Mohan M. Trivedi, Video-Based Lane Estimation and Tracking for Driver Assistance: Survey, System, and Evaluation, IEEE Transactions on Intelligent Transportation Systems, vol. 7, no. 1, March 2006, pp. 20-37.

[8]. Narayan Pandharinath Pawar \& Minakshee M. Patil, Driver Assistance System based on Raspberry Pi, International Journal of Computer Applications (0975 - 8887) Volume 95- No.16, June 2014, pp. 36-39.

[9]. R. Cucchiara, C. Grana, M.Piccardi\& A. Prati, Detecting moving objects, ghosts, and shadows in video streams, on Pattern Analysis and Machine Intelligence(PAMI), IEEE Transactions Vol. 25(10), 1337 - 1342, 2003.pp.25-28 


\section{IJIREEICE

[10]. S. Tuohy, D. O'Cualain, E. Jones, \& M. Glavin, Distance determination for an automobile environment using inverse perspective mapping in OpenCV, in Proc. Irish Signals and Systems Conference 2010.

[11]. Jaehyoung Yu, Youngjoon Han, HernsooHahn, An Efficient Extraction of On-Road Object and Lane Information Using Representation Method, IEEE International Conference on Signal Image Technology and Internet Based Systems, 2011.

[12]. E. Chou, Y. Sun, E.Salari, Automated Pavement Distress Detection Using Advanced Image Processing Techniques ,IEEE Proceedings 2009.

[13]. Awais Adnan, Saleem Gul, Muhammad Ali, Amir Hanif Dar, Content Based image Retrieval Using Geometrical Shape of Objects in Image, IEEE 2007.

[14]. OpenCV Python Neural Network Autonomous RC Car, http://github.com/hamuchiwa/AutoRCCar

[15]. Zheng Wang, Self Driving RC Car, http://zhengludwig.wordpress.com/projects/self-driving-rc-car/

\section{BIOGRAPHIES}

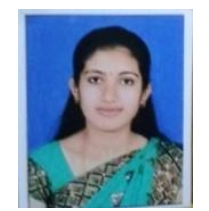

Mrs. Susmitha B C Assistant Professor, Electronics and Communication Dept., G Madegowda Institute of Technology, Bharathinagara (K M Doddi), Mandya

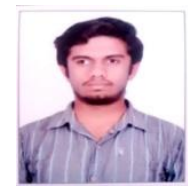

Mr. Karthik Sharma K 8TH Semester, Electronics and Communication Dept. G Madegowda Institute of Technology, Bharathinagara (K M Doddi), Mandya

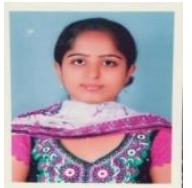

Ms. Pavithra N, 8TH Semester, Electronics and Communication Dept., G Madegowda Institute of Technology, Bharathinagara (K M Doddi), Mandya

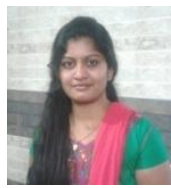

Ms. Nandini M R, 8TH Semester, Electronics and Communication Dept., G Madegowda Institute of Technology, Bharathinagara (K M Doddi), Mandya

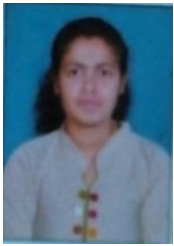

Ms. Punyashree M C, 8TH Semester, Electronics and Communication Dept., G Madegowda Institute of Technology, Bharathinagara (K M Doddi), Mandya 